\title{
Quand la révolution fait le mur : l'art mural vénézuélien entre imagination contestataire et contestation imaginaire
}

Popular iconography in Venezuela: in between rebel imagination and imaginary rebellion

\section{Fabrice Andréani}

\section{Q OpenEdition Journals}

\section{Édition électronique}

URL : http://journals.openedition.org/conflits/18799

DOI : 10.4000/conflits. 18799

ISSN : $1777-5345$

\section{Éditeur :}

CECLS - Centre d'études sur les conflits - Liberté et sécurité, L'Harmattan

\section{Édition imprimée}

Date de publication : 31 décembre 2013

Pagination : 123-139

ISBN : 978-2-343-02618-3

ISSN : $1157-996 X$

\section{Référence électronique}

Fabrice Andréani, «Quand la révolution fait le mur : l'art mural vénézuélien entre imagination contestataire et contestation imaginaire », Cultures \& Conflits [En ligne], 91/92 | automne/hiver 2013, mis en ligne le 31 décembre 2014, consulté le 31 mars 2021. URL : http://journals.openedition.org/ conflits/18799; DOI : https://doi.org/10.4000/conflits.18799 


\section{Quand la révolution fait le mur : I'art mural vénézuélien entre imagination contestataire et contestation imaginaire}

\section{Fabrice ANDREANI}

Fabrice Andréani est Doctorant en Science politique au CERI-Sciences-Po (Paris) et chercheur associé au CERAPS / Université Lille 2 Droit et Santé. Sa thèse porte sur la légitimation circulante de la " révolution" bolivarienne à l'international. Il a récemment publié "Du nomadisme idéologique à l'allégeance partisane: les mondes franco-vénézuéliens de la ré-élection de Hugo Chavez (2012) ", Critique internationale, $n^{\circ} 59,2013$, pp. 119-132.

"Un être sans études est un putain de policier"

Tag d'étudiants chavistes, Faculté de Médecine, Université des Andes, Mérida, 20071

T e 12 octobre 2004, le Paseo Colón de Caracas est le théâtre d'une " occuLation » peu ordinaire ${ }^{2}$. Des centaines d'artistes et d'intellectuels ${ }^{3}$ entendent honorer à leur manière l'appel du Comandante Hugo Chávez à la célébration du Jour de la Résistance Indigène, fête bolivarienne ${ }^{4}$ marquant l'arri-

1. «Un ser sin estudios es un maldito policia ». Posé devant cette « université bourgeoise » (autre tag des auteurs), il détourne le slogan «Un être sans études est un être incomplet » («Un ser sin estudios es un ser incompleto ») de Simón Bolívar, imprimé sur les murs de l’Université Bolivarienne du Venezuela - sensée faire concurrence à la première.

2. Je remercie vivement Mila Ivanovic et Xavier Crettiez pour leurs critiques des versions successives du texte. La représentativité des éléments empiriques mobilisés, dont je spécifie au cours du texte les conditions d'obtention, a été évaluée à l'aune de ma présence continue au Venezuela entre mai 2007 et juin 2009, puis entre juin et août 2010 - puis à distance grâce aux contacts noués sur place. Avec le photographe-reporter Mitia Rames Aptekier, nous avons notamment réalisé environ 1000 clichés d'images murales tant «sauvages » qu'officielles - à Caracas, à Mérida et dans les villages andins, sur la côte Nord-Est, et aux frontières andine, amazonienne et côtière d'avec la Colombie.

3. Essentiellement des artistes plasticiens et écrivains, des étudiants et des enseignants, ainsi que des travailleurs sociaux.

4. Nous déclinons sans guillemets bolivarien (étiquette non univoque), et avec guillemets " révolutionnaire "; en italique processus, synonyme de « révolution " bottom-up et par les urnes (la « voie chilienne » de Salvador Allende (1970-1973) distincte de la « voie cubaine », armée) et chavisme, ensemble mouvant, situé, d'allégeances au chef. 
vée des conquistadores en Amérique ${ }^{5}$ : ils mettent en scène un procès de Christophe Colomb, ou plus exactement de sa statue ${ }^{6}$. Après lectures des témoignages du prêtre Bartolomé de Las Casas dénonçant la sur-exploitation des amérindiens, le tribunal populaire déclare le navigateur « coupable du plus grand génocide de l'histoire humaine ». En file indienne, plusieurs dizaines de jurés tirent sur une corde mettant l'œuvre d'art au sol. Amarré à un pick-up, le condamné est traîné sur quatre kilomètres le long des principales artères capitolines, jusqu'aux abords du théâtre national Teresa Carreño où des milliers de partisans attendent patiemment l'arrivée de Chávez, maître des cérémonies officielles. Les art'ivistes ${ }^{7} \mathrm{y}$ infligent au mort un rite de purification indigène, préalable à son offrande au leader. Mais la police, alertée par des commerçants du paseo, devance ce dernier et disperse violemment la mobilisation, embarquant cinq présumés leaders et les morceaux de statue brisée. Si le soir même, Chávez désavoue à la télévision les « jeunes fauteurs de troubles », trois ans plus tard, le seul d'entre eux à comparaître en justice sera finalement relaxé ${ }^{8}$.

Aujourd'hui, qu'ils fassent ou non encore allégeance au chavisme, la majorité des acteurs de la révolution iconographique qu'a connue le centre-ville de Caracas au cours des années 2000 - à l'instar de la plupart des espaces publics du pays - considèrent le « procès de Colomb » comme leur baptême du feu ${ }^{9}$. Revendiqué de part et d'autre des frontières actuelles de l'officialisme 10, l'épisode reflète au mieux l'ambiguïté du processus bolivarien et de la place de choix qu'y occupent des arts de rue jadis marginalisés car jugés déviants ou subversifs, en particulier l'iconographie murale. Envisageant la production iconique de scènes "révolutionnaires » à partir de son contraire - la destruction de symboles « capitalistes »-, les «passeurs de limites » 11 officiels comme dissidents tracent une équivalence entre leur art et les occupations de terres, d'usines ou de logements. En l'espèce, tous voient dans l'issue de la manifestation d'octobre 2004 - la relaxe de leur « camarade »- une victoire de

5. À la demande d'organisations indigènes, le nom de l'ancien «Jour de la Race [métis] » associé au Columbus Day étasunien a été changé par décret-loi. Suite à leur adhésion à l'Alliance Bolivarienne pour les Peuples de Notre Amérique (ALBA-TCP) créée par Chávez et F. Castro (2004), la Bolivie, le Nicaragua et l'Équateur ont fait de même.

6. Le Monumento a Colón en el Golfo Triste (R. de la Cova, 1904), érigé en 1934 sous la dictature de Juan V. Gómez.

7. D’origine étasunienne, le néologisme signifie « artiste activiste ».

8. Les actes sont en ligne : http://caracas.tsj.gov.ve/decisiones/2007/mayo/1728-22-S7-3130-07S05-01.html (consulté le 15/02/2012).

9. Observations et entretiens, octobre 2007, mai 2008, février 2009 et juillet 2010. Et ce bien que la gauche locale n'ait pas le monopole de la destruction de statues : deux ans plus tôt jour pour jour, une même action à l'encontre d'un monument à l'effigie de C. Colomb était relevée à Barcelone (Espagne); au Venezuela, dans la foulée du "procès de Colomb », un groupe d'extrême-droite a fait éclater à coups de revolver une statue en verre du Che Guevara dans les règles de l'art paramilitaire - sans simulacre de justice avant exécution - sur le col du pico Ávila, haut lieu touristique de l'État andin de Mérida, frontalier d'avec la Colombie.

10. Le terme désigne le chavisme « officiel », soit l'ensemble des militants et élus occupant des fonctions publiques.

11. Terme désignant les hérauts des carnavals et autres rites de renversement symbolique de l'ordre social dans Balandier G., Le désordre : éloge du mouvement, Paris, Fayard, 1988. 
la « raison » plébéienne sur l'« ignorance » policière. Mais tandis que les uns attribuent le désaveu initial de Chávez au poids de la « droite endogène [au chavisme] » 12 , les autres y détectent la prémisse d'une «trahison » du mouvement social qui l'a porté au pouvoir, entérinée depuis par les héritiers du chef défunt, taxés en bloc de «boli-bourgeois ${ }^{13}$.

À l'aune de cette controverse, au Venezuela comme ailleurs, les iconographies publiques labellisées «contestataires » par leurs producteurs ou exégètes recèlent une capacité performative aussi puissante que versatile, à la différence des arts graphiques exposés dans des espaces clos. D'un côté, la transformation sensible de l'urbanisme - ici d' " ancien régime » - donne à voir in situ et in vivo une redistribution utopique des rôles et des ressources sur le territoire « repris » par les artistes. De l'autre, la visibilité même des œuvres, synonyme d'indifférence sinon d'aval gouvernemental, transforme à terme les attributs esthétiques du " changement» en gages du statu quo ${ }^{14}$. À cet égard, l'art mural des rues vénézuéliennes, fort d'une prolifération sans précédent ${ }^{15}$ et quasi-exclusivement le fait de chavistes d'extrême-gauche - « critiques »-, de libertaires ou d' «a-politiques », constitue un cas limite d'iconographie contestataire. Légalisé en tant que média "citoyen » dans une démocratie hyperpolarisée où la contestation est érigée en savoir d'État, il constitue un analyseur incontournable de la (dé)légitimation de la « révolution » bolivarienne ${ }^{16}$. À travers les luttes de classement et les (re)positionnements qu'il suscite au sein de milieux (contre)culturels redoutés par les élites bolivariennes pour leur relative indiscipline électorale ${ }^{17}$, se dessinent les potentialités et les limites inhérentes à tout entreprise artistico-politique, séditieuse ou étatique. Les comprendre implique néanmoins de ne pas réifier l'art en pratique exceptionnelle $18:$ l'analyse des images dans les configurations spatiales où elles sont

12. Bureaucrates, militaires, élus et cadres « infiltrés » ou transfuges de la Quatrième république (1958-1999).

13. Le terme "boliburgueses» («bourgeois bolivariens »), désigne indistinctement (hauts) fonctionnaires et entrepreneurs.

14. Sur cette ambivalence voir Rancière J., La nuit des prolétaires. Archives du rêve ouvrier, Paris, Fayard, 1981.

15. On peut raisonnablement parler d'une multiplication par 5 des productions - «sauvages » et officielles. Aujourd'hui, on compterait 5000 fresques pour 30 millions de passants. Observations et témoignages (2007-2009 et été 2010).

16. «Les actes de théâtralisation par lesquels les groupes se donnent en spectacle » en constituent la «forme élémentaire » in Bourdieu P., "La représentation politique. Éléments pour une théorie du champ politique ", Actes de la recherche en sciences sociales, 36-37, 1981, p. 11. Aussi les fêtes et rituels sont-ils des « formes de rationalisation du pouvoir » - Lagroye J., "La légitimation », in Grawitz M., Leca J. (eds.), Traité de science politique, I, Paris, PUF, 1985, p. 414.

17. De statuts intermédiaires ou de solidarités primordiales subalternes, les milieux (contre)culturels échapperaient aux logiques de polarisation régissant les autres secteurs (travailleurs informels, fonctionnaires, patrons « rouges " d'un côté ; employés privés, professions libérales, élites déchues de l'autre). Ellner S., "Venezuela's bottom-up participation: advances and downsides", Communication non-publiée, LASA Congress, 30, San Francisco, USA, 24 mai 2012.

18. Voir Rancière J., Le partage du sensible. Esthétique et politique, Paris, La Fabrique, 2000. 
exposées ne s'avère heuristique qu'en connaissance de leurs conditions sociales de production, de circulation et de réception ${ }^{19}$.

Située à la confluence des mondes de la guérilla et du hip-hop ${ }^{20}$, l'iconographie murale vénézuélienne recèle une charge subversive qui déborde de loin sa promotion gouvernementale face aux manœuvres putschistes dirigées à l'encontre du président Chávez (I). Partant, son assimilation à la « révolution » s'avère limitée, à l'image du travail d'artistes mandatés pour associer ses différents mondes aux campagnes électorales. Mettant leur temps libre et leurs dotations culturelles au service du régime, ils composent avec divers types d'illégalismes artistiques "a-politiques », dont la tolérance induit des effets contradictoires sur la légitimité et la cohésion interne du chavisme (II).

\section{Les arts-de-faire marginaux en " révolution »}

$\mathrm{Au}$ Venezuela, rares sont les publicités partisanes officielles qui ne soient pas environnées de tags, graffitis, pochoirs ou fresques aux accents révolutionnaires. Communément identifiée comme « chaviste » à droite de l'échiquier politique et objet de théorisations sophistiquées dans les cercles intellectuels bolivariens, cette imagerie suscite des usages contradictoires, du fait du brouillage des hiérarchies et appartenances sectorielles découlant des crises politiques qui l'ont rendue visible ${ }^{21}$.

\section{De la communication de guérilla à la " guérilla communicationnelle »}

Les inscriptions et scènes murales anticapitalistes sont d'abord la marque de fabrique des guérillas rurales et urbaines investies à partir des années 1960 par le Movimiento izquierda revolucionaria (MIR) et le Parti communiste (PCV), exclus du jeu politique pour cause de " guerre froide ", et qui ont perduré sous diverses formes jusqu'au début des années 1980 22. C'est ensuite l'importation de la contre-culture hip-hop des métropoles étasuniennes qui facilite la diffusion des techniques correspondantes par-delà ces cercles d'initiés. Alors que le contre-choc pétrolier de 1983 plonge le pays dans une crise de la dette sans précédent en Amérique 23, l'hybridation de ces deux mondes se nourrit de deux événements rétrospectivement jugés «fondateurs » de la révolution : le Caracazo de février 1989, émeute faisant suite à l'adoption d'un

19. Voir Balanskinski J., Matthieu L. (eds.), Art et contestation, Rennes, Presses Universitaires de Rennes, 2006.

20. Nous écrivons «monde » au sens de Howard Becker, « Retour sur les mondes de l'art », Propos sur l'art, Paris, L'Harmattan, 1999.

21. Voir sur ces mécanismes Dobry M., Sociologie des crises politiques, Paris, FNSP, 1983.

22. Une fois le MIR (issu du parti Acción democrática, alors gouvernant) et le PCV ayant accepté l'amnistie de 1968, le Partido de la revolución Venezolana de Douglas Bravo prendra, entre autres, le relais.

23. Le pétrole, dont le Venezuela est la première réserve mondiale, compose plus de $90 \%$ des exportations et $30 \%$ du PIB. Voir Coronil F., The magical State. Nature, money and modernity in Venezuela, Chicago, University of Chicago Press, 1997. 
plan d'ajustement structurel préconisé par le Fonds monétaire international (FMI) et « pacifiée » au prix de plus d'un millier de morts ; de l'autre, le putsch raté du Mouvement Bolivarien révolutionnaire (MRB-200) en février 199224.

Rendu célèbre pour avoir endossé à la télévision la « responsabilité » de son échec «provisoire " lors de son incarcération, le lieutenant-colonel Chávez gagne les présidentielles de 1998 à la tête d'une large coalition de gauche. Le 11 avril 2002, le président est mis aux arrêts suite à la la télé-diffusion d'un montage accusant ses partisans de tirer sur des manifestants d'opposition. Prises d'assaut par les putschistes, les télévisions publiques relaient la cérémonie d'investiture de Pedro Carmona, chef du syndicat patronal Fedecamaras, à la tête d'un "gouvernement de transition », avant de diffuser des dessins-animés 24 heures durant. En réaction, des millions de ses électeurs se passent un mot d'ordre, tant à coups de SMS que d'inscriptions murales : "descendre des barrios 25 », " exiger qu'on [leur] rende [leur] président ». Restitué à la faveur d'une mobilisation record, le désormais Comandante d'une « révolution pacifique mais armée » triomphe aussi d'une grève pétrolière de plusieurs mois (2002-2003) et d'un referendum de révocation à mimandat (2004). Il déclare la « guerre au latifundio médiatique ». On retrouve encore aujourd'hui, dans diverses configurations territoriales, urbaines comme rurales, populaires comme bourgeoises ou encore dans des quartiers administratifs tels Capitolio à Caracas, deux inscriptions symptomatiques de la participation populaire à cette entreprise hétéroclite :

«Quand les médias diront la vérité, les murs se tairont ».

«Murs blancs, cerveaux vides 26 ».

De l'avis du gouvernement, les limites posées à l'influence de médias privés ouvertement séditieux et la promotion des «médias communautaires » ou «alternatifs » tardent à donner les « résultats » escomptés ${ }^{27}$. Par contraste, la prédominance de l'imagerie révolutionnaire sur les murs publics fait l'objet de réflexions aux plus hauts niveaux. Dans la foulée de la ré-élection de Chávez (2006), qui met tant bien que mal le cap vers le «socialisme du XXIe siècle » et l'« État communal 28 », des cadres du Parti socialiste uni du Venezuela (PSUV)

24. Sur les éléments constitutifs de la crise politique ante- et post-bolivarienne voir Andréani F., «Du nomadisme idéologique à l'allégeance partisane : les mondes franco-vénézuéliens de la ré-élection de Hugo Chávez », Critique internationale, 59, 2013, pp. 119-132.

25. Quartiers populaires généralement situés sur les hauteurs des grandes métropoles.

26. "Cuando los medios digan la verdad, las paredes callaran ". "Paredes blancas, mentes vacías» [Traduction de l'auteur]. Observations (2007-2009, été 2010).

27. Notamment la Loi sur la responsabilité médiatique, et la fermeture du canal «putschiste » RCTV (2007), remplacé par la télé « participative » Tves («Tu te vois»). Entretiens avec plusieurs experts-praticiens de la " contre-hégémonie », Universidad Bolivariana \& Instituto Venezolano de investigación cientifíca, Mérida, Caracas, Paris (janvier 2009, juillet 2010, décembre 2011).

28. López-Maya M., Lander L., “Venezuela's presidential elections of 2006: towards 21st century socialism?”, in Eastwood J., Ponniah T. (eds.), The revolution in Venezuela: social and 
cherchent à fidéliser les producteurs d'images "sauvages », majoritairement non encartés. Ils lorgnent le dévouement des plus aguerris à assurer, via des ateliers auto-gérés et gratuits, la transmission des savoirs-faire aux « jeunots ». Conformément à une pratique propre aux établissements scolaires et mairies «patriotes » où officient des artistes locaux - anciens élèves, voisins -, certains réalisent des fresques à l'occasion d'événements tels la Copa América de football (2007) et le Bicentenaire de l'Indépendance (2010); mais aussi dans le cadre de politiques "participatives » d'aménagement des centre-ville. Cellesci sont identifiées à la « récupération » d'un espace public «bradé » aux grandes enseignes, déjà objet d'initiatives art-ivistes plus ou moins légales. $\mathrm{Si}$ ces créations, généreusement rétribuées, permettent aux artistes chevronnés de «faire descendre les gamins des barrios ", l'association des artistes au gouvernement se heurte à une série de contradictions.

\section{Des bombes de peintures entre kalachnikovs et narcotiques}

La veille des législatives de 2010, la gouverneure du Distrito capital Jacqueline Farrías, tenue guerrillera pour l'occasion, inaugure une «brigade de jeunes guérilleros de la communication ", composée de mineurs. Elle appelle collèges et lycées à accueillir ces formateurs en « critique des médias ». L'opposition dénonçant l'« embrigadement des enfants » ${ }^{29}$, le corps enseignant s'y refuse, tandis que la plupart des artistes s'opposent à toute « récupération ». Derrière une uniformité de façade, la « guérilla communicationnelle » recouvre différents " partages des espaces, des temps et des formes d'activité ${ }^{30}$ selon les groupes concernés. En témoignent leurs rapports différenciés aux armes et aux narcotiques ${ }^{31}$.

En outre, les membres du collectif «marxiste-léniniste guévariste » Alexis vive (Alexis Vit) prétendent perpétuer l'héritage guérillero du barrio du 23 de Enero. Ouvertement armés dans le cadre de « [leur] guerre au narcotrafic et au para-militarisme colombien» ${ }^{32}$, ils s'opposent aux tentatives gouvernementales de désarmement des citoyens non intégrés à la « milice » officielle. Ils cultivent la mémoire de leurs propres " martyrs », tombés sous les balles de francs-tireurs lors du coup de 2002 (dont Alexis), puis de règlements de compte en cascade. Ils multiplient leurs portraits, aux côtés du célèbre Simon Bolivar encagoulé ( $c f$. document 1). Faisant aussi peu l'unanimité dans leur

political change under Chávez, Cambridge, DRCLAS / Harvard University Press, 2011, pp. 131-154.

29. "Docentes crean comités para combatir "Guerrilla de la comunicación” ", El Universal, 22 avril 2010.

30. Rancière J., Le partage du sensible..., op. cit., p. 12.

31. La moyenne annuelle de morts par balle dépasse celle de l'Iraq. Bien plus répandue - et tolérée - que la marijuana (autant que celle-ci en France), la cocaïne est en parité pouvoir d'achat environ 10 fois meilleur marché qu'en Espagne. Observations (2007-2009, été 2010).

32. «La guerra al hampa». Tarragoni F., «Le peuple est dans la rue. Politiques d'un art populaire et pratiques contestataires dans le street art barrial au Venezuela », Communication nonpubliée (disc. Fabrice Andreani), J.E. « Penser la culture dans le Venezuela contemporain », 
quartier - bien que majoritairement " rouge »- que la kalachnikov mise dans les bras de la Vierge ou du Christ par des groupes voisins également issus de la guérilla ${ }^{33}$, la cagoule du Libertador symbolise la continuité entre la lutte estudiantine et proto-guerillera des années 1980, le néozapatisme chiapanèque, et l'actuelle " révolution dans la révolution ». Sur fond d'images du Caracazo (1989), elle rappelle aux «boli-bourgeois » qu'ils doivent « commander en obéissant 34 ». L' " avantgarde » conditionne son projet communautaire " intégralement autogéré ", Comuna el Panal 2021 35, à la garantie de son «droit » à « tenir le quartier ». À l'inverse, non armés, les Guerreros de la Vega

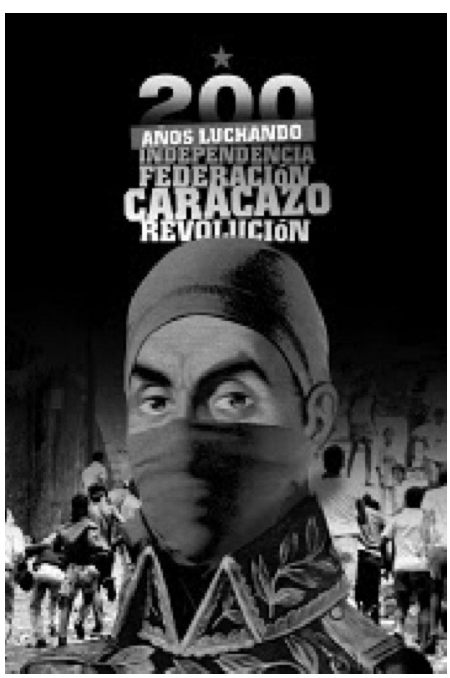

Document 1 - Simon Bolivar encagoulé (Alexis Vive, 2008) appellent à une « réconciliation nationale » abhorrée par les premiers - et voulue surtout à droite. Ils « [relayent] le message chrétien du gouvernement » via des fresques donnant «plus de chaleur » au slogan « vis la vie sans drogue » de l'Office National Anti-drogues ${ }^{36}$.

Issue aussi du 23 de Enero, mais déployant ses activités au centre-ville, l'Armée communicationnelle de libération (Ejercito comunicacional de liberación, ECL) revendique un « chavisme critique » à égale distance de ces deux types d'engagements. S'inspirant des créations de muralistes de la génération guerrillera - tels Nelson Santana ou Winstor Salas -, l'ECL se veut un espace de rencontre entre les compétences politiques de jeunes de classe moyenne ou « en voie de sortie du barrio » et les savoirs-faire propres au hip-hop. Autodidactes ou étudiants à l'Université expérimentale des arts (UNEARTE) - autonome -, ses formateurs usent de lieux procurés par des Conseils communaux pour leurs ateliers, mais refusent toute allégeance partisane. Ils conçoivent volontiers leur « agenda propre » comme étant « en marge » des champs culturel et académique, bien que leur publications, telles la revue Plomo (Plomb) et le livre Mural y Luces, soient appuyées par le Ministère du Pouvoir Populaire pour la Communication et l'Information ${ }^{37}$.

Groupe d'Études Interdisciplinaires sur le Venezuela, Paris, 28 juin 2012.

33. Tels les Tupamaros - qui est aussi un parti satellite du PSUV - ou le collectif La Piedrita.

34. "Mandar obedeciendo", consigne néo-zapatiste de l'EZLN du sous-commandant Marcos. La page d'accueil de leur site web déclenche un chant sandiniste (Nicaragua), et expose des liens vers ceux des FARC et de l'ELN (Colombie) : colectivoalexisvivecarajo.blogspot.com/

35. Nom évoquant les tags «Chávez 2021 » dans tout le pays, invoquant un droit « divin » à gouverner jusqu'à cette date.

36. "Viva la vida sin drogas»[Traduction de l'auteur]. Témoignages, documentaire « Murales y grafitis : los lienzos del pueblo » (2004). Produit par le Ministère de la Culture et diffusé sur Tves, mais montrant aussi des graffeurs très critiques vis-à-vis du chavisme, il a fait l'objet de censures répétées sur le web. 
Avec un titre détournant le slogan « Moral y Luces » ( morale et lumières ») de l' «éducation socialiste 38 », Mural y Luces (Mural et Lumières) promeut ce que José Roberto Duque a théorisé sous le néologisme « ouesthétique 39 » : l'omniprésence de l'esthétique chaotique des barrios pauvres, pour la plupart situés à l'Ouest de Caracas. Aux côtés de photos de murs et d'entretiens de membres de brigades informelles, des textes de vulgarisation para-académique attestent la confluence « révolutionnaire » de l'ensemble des traditions iconiques qu'ils y décèlent : agit-prop bolchévique, muralisme des révolutions mexicaine puis cubaine, situationnisme du « mai [68] français », propagande des guérillas chiliennes des années Allende, et graffiti hip-hop ${ }^{40}$. Au fil des pages, on décèle l'ambivalence de l'assimilation de l'ensemble des productions à une même «contre-hégémonie » : là où d'aucuns évoquent le «troc de revolvers et de drogues contre des bombes de peintures ", certains des graffitis dépeignent des caciques indigènes de l'époque coloniale dans des postures chamaniques, légitimant l'usage d'hallucinogènes.

\section{L'officialisme iconographique à la conquête de ses marges}

Fin 2010, le Ministère du pouvoir populaire pour les communes et la protection sociale (MPPCPS) entreprend de dédoubler l'ECL via l'institution d'une franchise "semi-autonome »: le Comando Creativo ${ }^{41}$. Ses membres, une cinquantaine, ont pour la plupart fait des études. À la différence des brigades informelles, le CC est sensiblement plus visible en période électorale. Son nom fait référence à celui des «QG » de campagne : en 2012, le Comando carabobo (PSUV et alii), et le Comando Venezuela (MUD : opposition). Or si les «manifestations »du premier sont, pour des fonctionnaires composant une bonne moitié des troupes, des séjours d'entreprise payés et obligatoires, celles de son faux-frère " créatif » reposent sur le volontariat : moins d'une demi-douzaine d' " anciens » y travaillent pour le MPPCPS, et le restant sont bénévoles ${ }^{42}$. À la lumière du récit d'une militante tardive, les vertus (auto)dis-

37. Ils sont en cela comparables aux collectifs analysés dans Geoffray M-L., Contester à Cuba, Paris, Dalloz, 2011.

38. Attribué au maréchal Antonio José de Sucre, compagnon d'armes de Simón Bolívar. C’est le « 3ème moteur de la révolution » dans la « nouvelle géométrie du pouvoir » (2006). L’Université Bolivarienne s'appelle aussi Misión Sucre.

39. La oestética. Jarman R., «Del este al oeste. La producción topográfica y la diferencia sociocultural en Caracas : Transformaciones socio-geográficas desde 1989 », Communication nonpubliée (disc. Fabrice Andreani), J. E. «Penser la culture... », op. cit.

40. Notamment: Guaglianone J.L., «Arte urbano, culturas urbanas, y guerrilla simbólica. Una posible brújula para el arte visual urbano en Caracas », in ECL, Mural y Luces, Caracas, ECL/MPPIC, 2010.

41. Sur le CC, des entretiens et observations ont été réalisés à Caracas en juin et juillet 2010. Pilar, rencontrée en octobre 2007, mai 2008, février 2009 et juillet 2010, se soumet, de juin à novembre 2012, à des entretiens à distance - par Skype, à raison d'un par mois ; elle fait alors circuler une dizaine de questionnaires.

42. Les cadets exécutent parfois des commandes, mais pour " arrondir les fins de mois ». On retrouve notamment au CC des agents contractuels ou vacataires d'autres administrations (parapubliques - travailleurs sociaux, instituteurs, enseignants), des actionnaires de coopératives, des travailleurs indépendants, et des étudiants boursiers. 
ciplinaires de leur engagement, adossé à une situation socio-spatiale intermédiaire, trouvent leurs limites dans l'illégalisme structurant l'ordinaire métropolitain.

\section{Une campagne "créative »}

Doctorante en Lettres à la prestigieuse Université centrale (UCV), Pilar, 26 ans, a intégré le CC début 2012. Employée dans une librairie, elle était jusque là taxée d' "anarcho-bourgeoise " par ses amis chavistes: elle a voté contre la révocation de Chávez en 2004, mais s'est abstenue en 2006, et a refusé la réforme constitutionnelle de 2008, comme sa forme allégée l'année suivante - légalisant la réélection illimitée du président. De parents «issus du barrio » et devenus médecins, elle se défend de venir d'une «famille friquée »: si elle a acquis la nationalité espagnole, c'est grâce à « l'origine humble » de ses grands-parents paternels, réfugiés du franquisme. D'ascendance iranienne bahaï côté maternel, elle a longtemps «fait du social » en colonie, mais refusait sa « récupération» par les "alliés » d'un régime islamique qui persécute les «siens ». C'est de retour d'un voyage à Cuba qu'elle franchit le pas, non par fascination pour le «socialisme » mais, au contraire, au vu de la «misère » de l'île, dont la « dignité » des habitants l'amène à « relativiser » ses propres " coups de blues». Elle fait part de ces réflexions à Kael, co-fondateur du CC, et ami du lycée, entre deux cours communs à la UCV :

«Je faisais [alors] déjà des pochoirs, mais seule... des thèmes littéraires... Kael m'a dit qu'ils donnaient des ateliers de formation à la communauté, que je pourrais leur filer un coup de main... J'ai commencé par les pochoirs, à Catia TV [communautaire]. Après ils ont ouvert un atelier de fresques. C'était dément, on a occupé des murs énormes, on mélangeait acrylique, pochoirs, calques, images vidéo, des patrons faits main... Puis j'ai organisé des cinés-forums ».

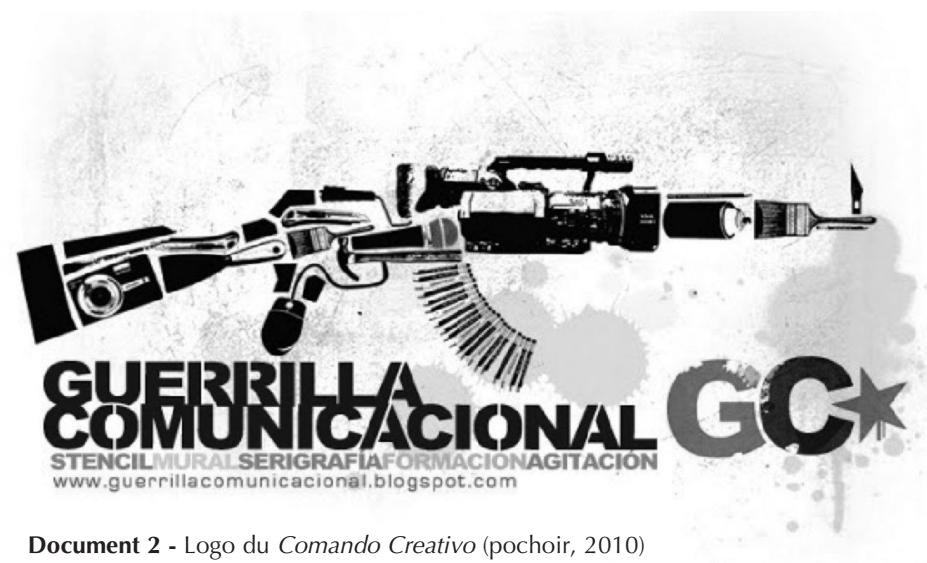


À l'instar du pochoir-logo le plus connu du CC (cf. document 2) ${ }^{43}$, posé sur tout type de mur des quartiers à la fois populaires et de classe moyenne d'un centre-ville en voie de gentrification, la " guérilla » dans laquelle Pilar s'engage mobilise les beaux arts, mais aussi l'audiovisuel, dont elle est déjà familière. Si elle conçoit que les apprentis puissent à terme "monter euxmêmes leurs images ", il faut d'abord qu'ils sachent "graffer debout plutôt que de peindre à genoux ${ }^{44}$ ( $c f$. document 3$)$. Tout en pointant leurs origines modestes et la satisfaction morale qu'elle en tire, Pilar dresse une équivalence entre sa propre «émancipation » et la leur :

« Au lieu de continuer à vouloir changer le monde dans mon coin, je suis sortie le faire en collectif... N'importe qui peut participer même s'il n'a jamais pris un pinceau... Beaucoup des ateliers qu'on donne, c'est à des gens qui veulent faire ça dans leur quartier, c'est pas des gens qui ont fait des études supérieures ou quoi... Y'a un mec qu'est mécano. Des jeunots. Des vieux aussi ».

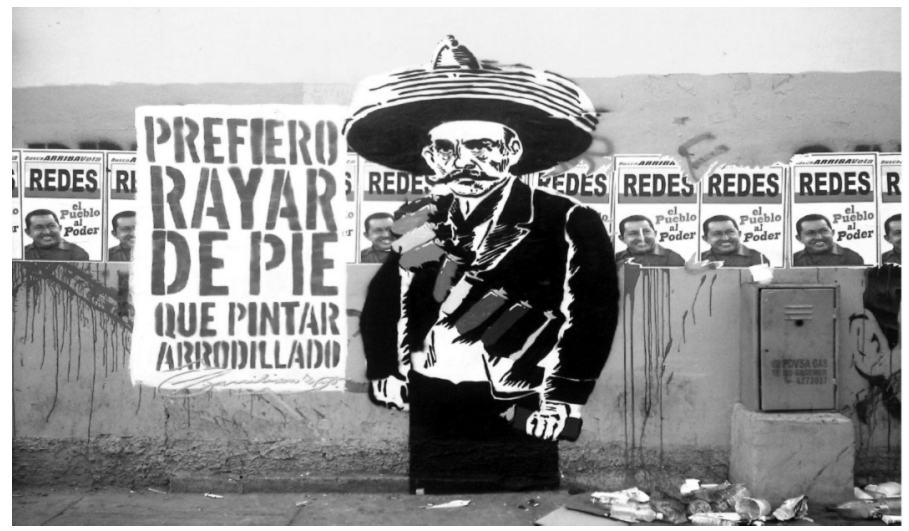

Document 3 - Slogan du Comando Creativo (pochoir, 2010)

L'entreprise impliquant la disponibilité de formateurs non rémunérés, Pilar insiste sur l' « autonomie » du CC vis-à-vis des financements « bureaucratiques ». Le CC travaille au besoin sur fonds propres, grâce à l' " inventivité » de ses membres. Celle-ci repose à la fois sur une culture universitaire et militante, et sur la maîtrise de l'abécédaire du travailleur informel dans cette « jungle urbaine » qu'est Caracas. Comme le précise Pilar, les périodes de vache maigre favorisent ainsi le lissage subjectif entre les conditions d'existence - et d' « émancipation »-des participants permanents et intermittents :

43. Repris notamment au Mexique et au Pays basque, il compose un fusil AK-47 en agrégeant : un marteau, un appareil photo, une palette, une souris d'ordinateur, un micro, des cartouches d'encre, une caméra, des bombes de spray et un pinceau.

44. Le slogan "prefiero rayar de pies que pintar arrodillado» détourne une phrase de Zapata lors de la Révolution mexicaine : "Je préfère mourir debout que vivre à genoux » ("prefiero morir de pies que vivir arrodillado »). 
«Le Ministère envoie du matériel, des fonds, mais on fait aussi avec nos propres moyens : on a sérigraphié des tee-shirts d'Ali Primera [chanteur pro-guérilla des années 1960-70], de Frida [Kahlo], de Bolivar avec des lunettes, de Chávez... On les a vendus dans la rue, pour acheter un groupe électrogène, un vidéo-projecteur... Car la bureaucratie de la Quatrième [République] est encore là, même au Ministère des "Communes"... On ne veut pas en dépendre. S’ils débloquent des fonds, les projets visés deviennent prioritaires, mais pour le reste on est autonome... »

C'est justement une fois lancée la campagne de 2012 qu'abondent les fonds publics, facilitant les mobilités à travers le pays. Un QG « alternatif » s'installe en dehors de Caracas, chez des artistes et intellectuels d'obédience "libertaire ", les Cayapos ${ }^{45}$. Le pochoir conçu avec leurs pairs de Hip-bop Revolución (cf. document 4), collé quant à lui sur des murs plus «bourgeois » que de coutume, tels ceux des centres commerciaux, illustre la philosophie à l'œuvre dans cette émulation "trans-classiste " ${ }^{46}$. Y sont listés et revendiqués une dizaine de stigmates associant déviances morales et politiques, pour conclure sur une profession de foi : "nous autres [ie : les "marginaux"] avec Chávez ${ }^{47}$. Pilar raconte :

«Les cayapos sont des vieux hippies qui ont une Commune vers Valencia. Ils expérimentent des formes de vie alternatives sur un terrain occupé : ils ont construit des maisons avec du matos récupéré, un grand potager... Ils sont artistes de cirque, profs, danseurs, photographes, écrivains, alcooliques... On a organisé des sortes de colos : on a amené plein de monde, pour planifier une campagne alternative [à celle du GPP], en remplissant des murs de consignes atypiques: “on est des gens qui ne veulent plus être dirigés". J'ai donné un atelier de pochoirs aux gars de Hip Hop Revolución. On a fait des micro-docus... Ces vieux m'ont fait voir un autre monde. »

Pilar et ses amis associent ces échanges de savoirs-faire, l'intensification des cadences productives, et l'émergence de nouveaux projets. L'escompte du « résultat » final confère une dimension mystique à leur travail. L'apparition publique du Comandante quelques jours avant le scrutin est synonyme de

45. Dont des penseurs de l'« ouesthétique ». La cayapa est une tradition paysanne de communisation du travail manuel - chantiers d'habitations, routes, champs -, à l'instar des « journées chinoises » chez les « soixante-huitards ».

46. Pour parler comme le philosophe du «bon populisme », Ernesto Laclau - cité par nombre d'intellectuels chavistes.

47. «Depuis le pouvoir on nous a traités : “Racaille. Tourbe. Scélérats. Bourrés. Flemmards. Tire-au-flanc. Vaux-riens. Puants. Négligés. Singes. Sales. Macaques. Lumpen. Chienlit. Voyous. Gamins. Cul-terreux. Chavistes”. Aujourd'hui nous sommes l'horizon. Nous autres avec Chávez ». 


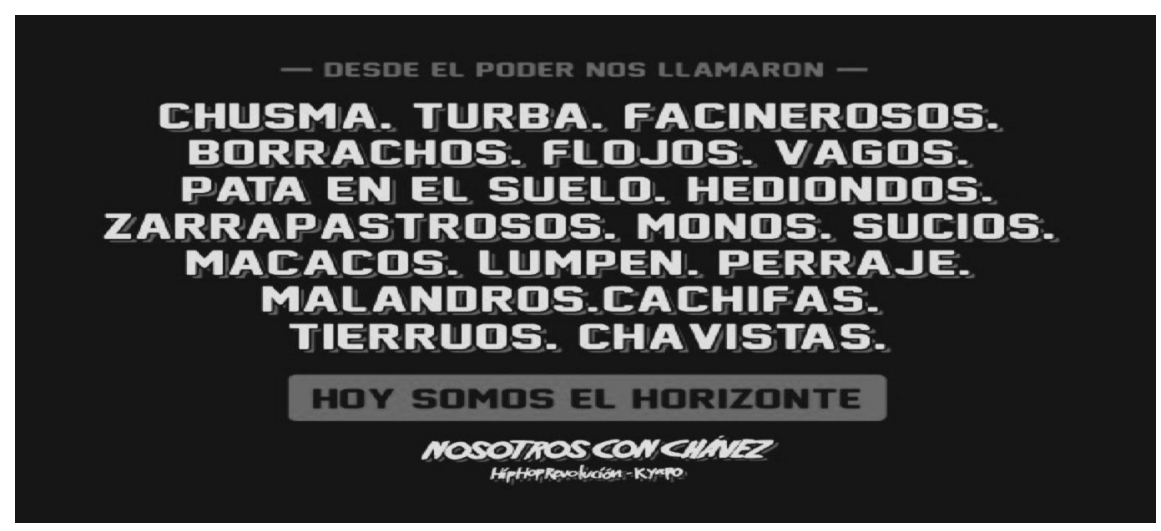

Document 4 - Campagne des collectifs Cayapo et Hip-Hop Revolución (pochoir, 2012)

reconnaissance de leurs efforts, légitimant une euphorie collective qui paraît d'autant plus sincère qu'elle n'est pas monnayée par l'octroi de quelque prébende que ce soit. Rétrospectivement, ils s'approprient la victoire de Chávez, et avec elle «leur rue », alors inondée, au sens propre comme figuré. Pilar se rappelle :

\begin{abstract}
"Ça a culminé avec la campagne... Le CC a collaboré avec 30 fresques, 300 t-shirts, des ateliers dans plusieurs États. Une autre brigade s'est formée dans le Falcón. Des gens de partout sont venus à Caracas pour l'atelier de muralisme, qui a duré 3 mois... On veut aussi faire une sorte d'école... Le jour de la clôture de la campagne c'était la folie. On avait fait des pancartes et des t-shirts et on est sortis dans la rue avec les pochoirs et tout le bordel, il pleuvait, c'était complètement fou, et Chávez est sorti à son balcon et on criait tous comme des dingues...»
\end{abstract}

\title{
Intellectuels et bons sauvages
}

Après la campagne, le retour à l'ordinaire donne cours à une plus grande liberté artistique. Ayant abandonné un temps certaines idées « de faible portée » électorale, Pilar met alors sa polyvalence technique, source d'intégration rapide mais acquise à force d'études et de voyages, au service de l'épanouissement de «sa [propre] culture politique », plus cosmopolite. Après s'être limitée à mettre une touche d'humour à des lieux communs électoralistes, tel celui du candidat d'opposition «impérialiste » ( $c f$. document 5$)^{48}$ fustigé comme tel

48. Ayant fait campagne sur sa « jeunesse » et sa « vigueur », et au physique avantageux, Capriles découvre la veste du survêtement national aux J.O., qu'il a coutume d'arborer, pour laisser entrevoir le drapeau étasunien. Ses oreilles de Mickey Mouse évoquent un jeu de mots usé par Chávez sur son deuxième nom de famille. Plutôt que « Henrique C. Radonsky », il l'appelle «H. C. Ratonsky» : le raton est la «souris » de Walt Disney. 
sur plus d'une centaine de murs du centre-ville, elle fait d'une figure des Black Panthers, aujourd'hui enseignante à UCLA, une porte-parole des « afros » du Nord comme du Sud (cf. document 6) ${ }^{49}$ :

«Demain [2 semaines après la victoire du 7 octobre 2012], on a une journée de pochoirs, graffs et peintures collectives. Je ferai Angela Davis, je suppose que tu la connais, la militante afro-américaine... J'ai fait mes premiers travaux en campagne. Du coup là je me lâche un peu... ${ }^{50}$ »

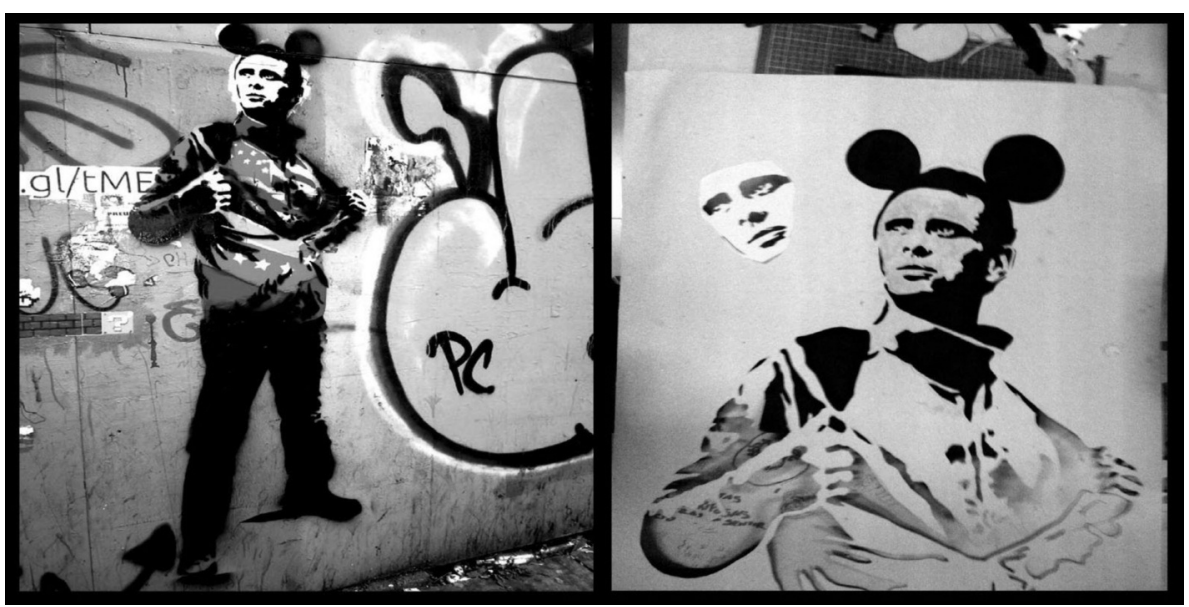

Document 5 - H. Capriles, principal candidat d'opposition à Chávez (CC, pochoir, 2012)

Ainsi « capable de s'adapter ", Pilar évoque avec auto-dérision, au vu de son expérience cubaine, la figure de l'« homme nouveau » théorisée par le Che : elle est «manuelle » autant qu' « intellectuelle». Or si on lui dit qu'il lui faudrait alors aussi savoir «manier le fusil » 51 , elle ne prend pas la plaisanterie à la légère. Hostile à l'usage d'armes à feux, elle ne souhaite pas "défendre la révolution » par cette voie-là. L'encourager, comme le groupe Alexis vive, ferait le jeu de l'opposition, qui amalgame engagement « radical » et banditisme. Déjà braquée au revolver, et «sauvée » par un chauffeur de taxi qui a abattu

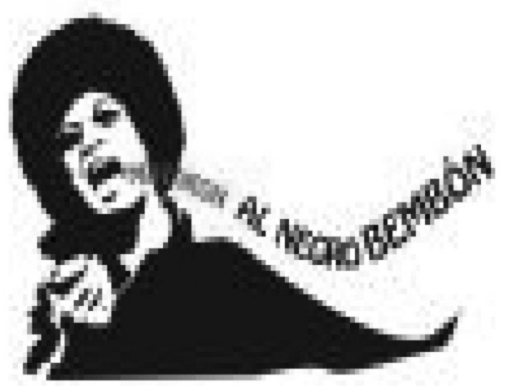

Document 6 - Angela Davis chante Ismael Rivera (CC, pochoir, 2012)

49. A. Davis y chante un refrain célèbre du salsero Ismael Rivera, "Mataron al negro bembon » (salsa) - « On a tué le noir aux grosses lèvres » (1954) [Traduction de l'auteur].

50. Entretien avec Pilar par Skype, 21 octobre 2012.

51. À l'image de la devise du collectif andin Victor Jara - chanteur chilien assassiné en 1973 : «estudio, trabajo, fusil». 
l'agresseur sous ses yeux, elle évoque aussi le passé d'un des apprentis, et ses stigmates. L'officialisme de sa militance autorise d'ailleurs des sorties d'ordinaire « risquées » :

"Comme uniforme, on porte un petit brassard. Du coup, si on sort en pleine nuit, personne ne nous emmerde. Ça c'est super, un voyou ne cherche pas des gens avec des brassards... Et puis un des gars [du $\mathrm{CC}$ a été bandit, il a sept entailles de couteau au cou ! »

En outre, les liens unissant violence armée et circulation de drogues illicites compliqueraient l'intégration des « marges » du chavisme. N'ayant jamais fumé de marijuana ou pris de cocaïne, Pilar dit respecter ces mours de « certains muralistes »- en fait une franche majorité -, en vertu de son éthique babaï. Ce «à condition qu'ils soient clean au boulot », " productivité » oblige, mais aussi pour ne pas véhiculer une « mauvaise image » aux passants.

De fait, c'est surtout à l'aune de son rapport instrumental aux publics profanes que cette « idéologie du graffiti $\$ 52$ tranche avec celle de nombre d'artistes informels. Las d'être taxés par les officialistes d' individualistes petitbourgeois » ou par leurs opposants de " gangs » vecteurs d'une «pollution visuelle qui exclut les bonnes gens », une dizaine de graffeurs hip-hop des plus réputés ont organisé une contre-offensive en images. Leur documentaire, Pinto con lata ${ }^{53}$ alterne ainsi propos anti-graff ${ }^{54}$, auto-justifications et virées nocturnes. Il révèle, en creux, les limites de la normalisation de leurs arts-defaire, auxquels ils dénient toute portée ${ }^{55}$ :

Senk 56 : «Ils se sentent exclus ? On est exclus d'un million de choses... Prends des gens lambda et montre leur un Miro, ou un Picasso pour faire simple. Combien pigent son délire de la quadruple-dimension ?... et combien applaudissent ? Moi, ça ne m'intéresse pas de "changer le monde". "

Dunk : «C'est un truc d'élites, entre les scientifiques, y'a un langage commun, c'est pareil... C'est pas un "moyen d'expression". "

52. Lachmann R., « Le graffiti comme carrière et comme idéologie », Terrains et travaux, 5, 2003, p. 55-86.

53. "Je peins avec des cannettes [de peinture] " [Traduction de l'auteur] : www.pintoconlata.com.

54. Recueillis auprès des publicitaires, urbanistes, architectes et habitants des quartiers où ils opèrent.

55. Là où « chaque culture prolifère par ses marges », bien qu'elles sont des « exceptions » dans "l'imaginaire officiel », de Certeau M., La culture au pluriel, Paris, Seuil, 1993, p. 213.

56. Les noms à gauche sont les signatures des graffeurs. Les propos sont issus du film Pinto con Lata. La réputation des auteurs est confirmée par des observations et témoignages in situ, en juin et juillet 2010, puis par les entretiens avec Pilar (juin à novembre 2012). 
Presque tous encagoulés à l'écran, ils louent une clandestinité génératrice de savoir-faire, comparables à ceux des (vraies) guérillas. Considérant la violence comme inévitable, ils jouent avec l'ambivalence de l'intérêt des « intellos » pour le bravo pueblo 57 - ces « classes dangereuses » :

Apl : «Mon jeu implique qu'on m'attrape. Si tu joues au gendarme et au voleur, on va pas te filer une carte disant "ne le touchez pas". " Senk : "Faut savoir déjouer les règles, quand et où passent les patrouilles... C'est une danse, un sport... Pas comme l'intello au chapeau sur le paseo... Avec le délire légal, t'es comme un artiste dans son atelier. »

Tuker : «Se faire un nom, occulte, en hauteur... Quand je laisse des traces, la rue m'appartient... Si tu me files une place "réservée", j'irai à côté... Tout l'espace doit être pris! »

Impe : «Le "sac de la ville”, on peut pas l'embastiller... C'est le mouvement, incognito, de nuit ».

Daos : «Ça génère des problèmes qu'on doit résoudre dans la rue... Les tocards, ils ont qu’à faire des murs légaux! »

Aussi comparent-ils leurs techniques d'occupation des murs avec celles de la publicité, assimilée au graff légal, et dont ils critiquent l'«immoralité » :

Alien : «Le graff légal, c'est des marchands de culture !».

Daos : «J'aime pas ces marchandises, je suis pour l'informel... Le spectacle, c'est cher payé... Le graff est arrivé à moi, je l'ai pas acheté dans un centre commercial. »

Apl : «Les pubs te mettent une nana seins nus au milieu de l'autoroute ... Et ça c'est légal parce qu'ils ont payé ? Le graff c'est pareil, si ça te plaît pas t'as qu'à l'ignorer. »

Senk : «Du “vandalisme” ? C’est ridicule, j’ai tué personne !... C'est bien de s'attaquer aux pubs... Mais pas pour promouvoir d'autres mensonges. »

Seuls à paraître à visage découvert, deux d'entre eux sont favorables à la coexistence pacifique entre graffiti illégal et légal - chaviste -, à condition d'un respect mutuel. Formateurs occasionnels et interviewés dans Mural y Luces, ils se « dédoublent » selon les circonstances :

Uki : «Y'a aucune norme qui tienne, c'est “anarchiste”... Je peux graffer dans mon quartier, les gens vont kiffer, ceux qui graffent quoi... Mais si je peins une fresque, les gens [les autres] vont encore plus kiffer...»

57. Comme dans l'hymne national (Gloria al bravo pueblo) et les pubs chavistes, ce « peuple » est à la fois « téméraire » et "énervé ». 
Dinamo : «Je peux graffer illégal la nuit, puis me pointer le matin à un atelier pour leur apprendre à faire une Vierge Marie! ».

Ceux-là font valoir leur droit au non engagement à l'écran. Devant un mur chaviste de la UCV, ils posent leurs « blases » sur une publicité surplombant l'autoroute d'une dizaine de mètres ${ }^{58}$, avant d'ajouter une phrase associant fétichismes marchand et idéologique: « mangez mes restes $\$ 59$. Ailleurs, ils complètent le slogan de Pepsi-Cola : "partagez... les rêves» 60 . Aussi leur «collaboration » avec le chavisme est-elle l'occasion d'instiller dans les espaces concernés un « discours caché » 61 sur la politisation de leur art. Entre autres, deux murs leur ont été «offerts » dans le cadre du Bicentenaire de l'Indépendance, entre l'Assemblée nationale et la Place Bolivar. «Intégrés » à une esthétique chaviste, leurs murs se distinguent par l'absence de symboles politiques, conférant une portée critique aux scènes dessinées. Sur l'un, le graff "Concreto ", aux lettres en forme de pavés, évoque un engagement " concret » et/ou «béton »; et réclamant aux passants d'être dissocié de celui d'artistes visiblement " révolutionnaires », souvent soupçonnés d'être " achetés " (cf. document 7). Sur l'autre, en associant leur ville à ses symboles les moins connotés idéologiquement, le métro et le lion, le graff «Yo soy Caracas 》 ( Je suis Caracas ») revendique un graffiti omniprésent et qui ne peut, par essence, être régulé ( $c f$. document 8$)$.

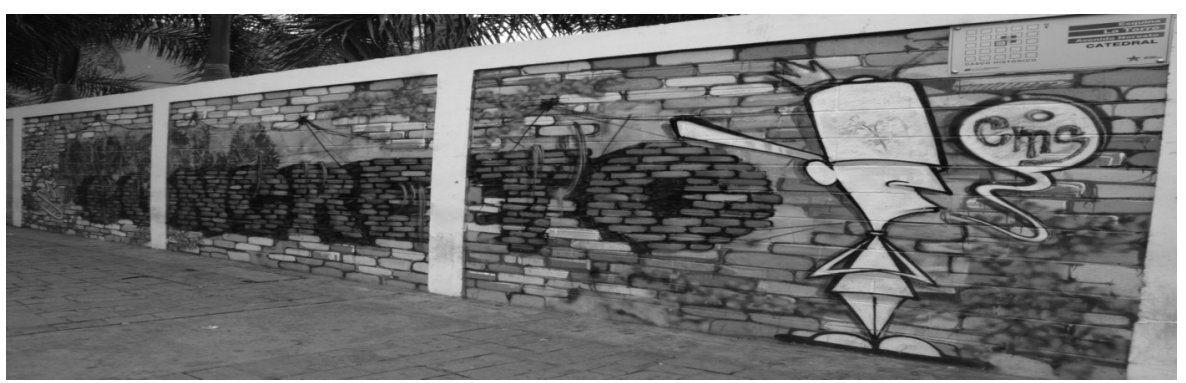

Document 7 - «Concret » / « béton » (graff, CMS, 2010)

58. Les risques s'amplifient avec la saturation murale : « les jugements sur la qualité artistique des œuvres sont liés à la quantité d'espaces disponibles pour les montrer ", H. Becker, op. cit., p. 113.

59. "Comanse mis sobras» [Traduction de l'auteur].

60. «Comparten ... las hipnas» [Traduction de l'auteur].

61. Scott J.C., La domination et les arts de la résistance. Fragments $d u$ discours subalterne, Paris, Éditions Amsterdam, 2008. 


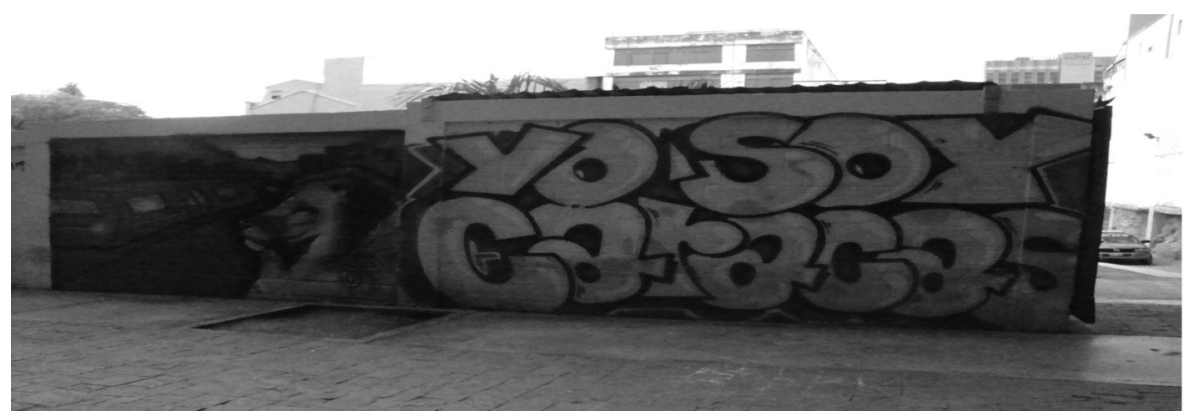

Document 8 - " Je suis Caracas » (graff, CMS, 2010)

La scène illustre bien la complexité des relations entre la pérennisation de l'ingénierie partisane chaviste et la persistance, voire l'augmentation, de différentes formes d'illégalisme populaire, irréductibles les unes aux autres. En fait de contestations, les élites chavistes doivent ici louvoyer entre les justifications post-guerilleras d'une violence politique incontrôlée et aux allures de guerre de gangs, et les éloges clandestins de "l'art pour l'art ». Usant des symboles des uns sans pour autant les désarmer, elles ménagent avec l'indifférence politique des autres. Bénéficiaire $\mathrm{du}$ « sens de la transformabilité politique du monde » 62 véhiculé par ces subversions larvées, ce «gouvernement des mouvements sociaux » 63 est aussi largement tributaire de l'informalité (statutaire, policière) qui les régit, les artistes les plus « marginaux » pouvant jouer ici et là - non sans ironie - les faire-valoir « populaires » du chavisme dans la rue. Ses représentants sont ainsi pris dans un jeu complexe entre le recrutement d'intellectuels et d'artistes sommés de s'engager au nom de leur statut, et la tolérance d'une critique subalterne croissante, jusqu'ici circonscrite à des expressions a-partisanes.

62. Cefaï D., Pourquoi se mobilise-t-on ? Les théories de l'action collective, Paris, La Découverte, 2007 , p. 526.

63. L’expression, « importée » de Bolivie, est du vice-président Álvaro García Linera, sociologue « marxiste indianiste ». 